\title{
Atribuições dos Técnicos em Saúde Bucal na Estratégia Saúde da Família em Minas Gerais, Brasil
}

\author{
Responsibilities of Oral Health Technician \\ in the Family Health Strategy in Minas Gerais, Brazil
}

Carla Aparecida Sanglard-Oliveira ${ }^{1}$

Marcos Azeredo Furquim Werneck ${ }^{1}$

Simone Dutra Lucas ${ }^{1}$

Mauro Henrique Nogueira Guimarães de Abreu ${ }^{1}$

${ }^{1}$ Departamento de Odontologia Social e Preventiva, Faculdade de Odontologia, Universidade Federal de Minas Gerais. Av. Antônio Carlos 6627, Pampulha. 31.270-901 Belo Horizonte MG. maurohenriqueabreu@ ig.com.br

\begin{abstract}
The scope of this article is to analyze the self-reporting of duties performed by the Oral Health Technicians in the State of Minas Gerais. A cross-sectional descriptive study was conducted through telephone interviews, with a representative sample of 231 workers. The descriptive analysis involved the calculation of proportions. It was found that 71.6\% (95\% CI, 64.4 to 77.5) performed coronal polishing, 63.2\% (95\% CI, 56.1 to 69.7) carried out scaling of dental calculus and $14.7 \%$ (95\% CI, 10.3 to 15.4) inserted restorative materials. Regarding preventive/collective actions, 100\% (95\% CI, 97.6 to 100.0) participated in educational activities, 99\% (95\% CI, 96.1 to 99.8) demonstrated oral hygiene techniques, 96.6\% (95\% CI, 92.7 to 98.4) administrated topical fluoride, $77.9 \%$ (95\% CI, 71.5 to 83.3 ) made home visits, and $96.6 \%$ (95\% CI, 92.7 to 98.4) performed collective actions, especially in schools. Oral Health Technicians have spent their more time on preventive/collective activities than on individual clinical care.
\end{abstract}

Key words Dental assistants, Human resources, Dental hygienists
Resumo O objetivo deste artigo é analisar o autorrelato de funções executadas pelos Técnicos em Saúde Bucal no Estado de Minas Gerais. Estudo transversal e descritivo, realizado por meio de entrevista telefônica, com amostra representativa de 231 trabalhadores. A análise descritiva envolveu cálculo de proporções. Observou-se que 71,6\% (IC $95 \%, 64,4-77,5)$ realizavam polimento coronário, $63,2 \%$ (IC 95\%, 56,1-69,7) faziam raspagem de cálculo e 14,7\% (IC 95\%, 10,3-20,4) condensavam e inseriam materiais restauradores. Em relação às ações preventivas/coletivas, 100\% (IC 95\%, 97,6-100,0) participavam de ações educativas, 99,0\% (IC 95\%, 96,1-99,8) demonstravam técnicas de higiene bucal, 96,6\% (IC 95\%, 92,7-98,4) realizavam a aplicação tópica de flúor, 77,9\% (IC $95 \%, 71,5-83,3)$ realizavam visitas domiciliares, $e$ 96,6\% (IC 95\%, 92,7-98,4) realizavam ações coletivas, principalmente em escolas. Os Técnicos em Saúde Bucal têm desprendido seu tempo mais em atividades preventivas/coletivas do que em atividades assistenciais.

Palavras-chave Auxiliares de odontologia, Recursos humanos, Higienistas dentários 


\section{Introdução}

Os sistemas públicos de saúde têm incorporado, há várias décadas, recursos humanos de nível técnico com o objetivo de aumentar sua produtividade e qualidade e visando mudanças no processo de trabalho e na produção do cuidado. $\mathrm{O}$ Técnico em Saúde Bucal (TSB) é uma das ocupações auxiliares do Brasil no âmbito da saúde bucal. Como as demais categorias auxiliares, essa ocupação foi criada com o objetivo de racionalizar o trabalho e aumentar sua produtividade e qualidade, visando ao desenvolvimento científico-tecnológico e às mudanças nas práticas e nos modelos de assistência. Historicamente, a incorporação desses trabalhadores permitiu tanto o aumento da cobertura, quanto a modificação no quadro de recursos humanos dos serviços de saúde ${ }^{1-4}$. Eles são atores importantes para a promoção, prevenção e recuperação da saúde bucal, junto ao sistema público ou privado, em nível individual ou coletivo ${ }^{2-4}$.

No Brasil, a primeira proposta para regulamentar o pessoal auxiliar foi o parecer 460 de 1975 do antigo Conselho Federal de Educação. Em 1984, o Conselho Federal de Odontologia CFO reconhece esses profissionais, disciplinando o exercício dos mesmos. Mas somente no ano de 2008, houve a publicação da Lei Federal no 11.889, descrevendo as atribuições e as restrições dos TSB no âmbito da saúde bucal ${ }^{5,6}$. Diversos movimentos democráticos a favor da utilização de pessoal auxiliar na odontologia aconteceram na década de 1980, em contraposição a interesses corporativos que se contrapunham a essa utilização. Assim, a profissionalização do TSB (anteriormente denominado Técnico em Higiene Dental - THD), bem como o rol de atribuições permaneceram comprometidos, durante a longa história de consolidação dessa ocupação no Brasil. Enquanto as atividades de uma determinada profissão não são parametrizadas por dispositivos legais, prevalece um espaço aberto e isento de princípios balizadores que estabeleçam o perfil para seu preenchimento ${ }^{7}$.

Atualmente, os TSB são importantes por compor as Equipes de Saúde Bucal que estão inseridas na Estratégia Saúde da Família (ESF), criada a partir da consolidação do Sistema Único de Saúde (SUS) no Brasil ${ }^{8}$. As Equipes de Saúde Bucal (ESB) inseridas na ESF podem ser formadas pelo Cirurgião-Dentista $(\mathrm{CD})$ e pelo Auxiliar de Saúde Bucal (ASB), sendo denominada de equipe modalidade I, ou pelo CD, ASB e TSB, denominada de equipe modalidade II. O traba- lho dessas equipes se baseia nos princípios da Atenção Primária à Saúde ${ }^{9}$, constituindo uma estratégia de organização de seus serviços no país ${ }^{10,11}$. No entanto, uma minoria de equipes de saúde bucal no Brasil é da modalidade II. Em abril de 2011, segundo dados do Ministério da Saúde, dentre as 20.640 ESB implantadas no Brasil, 1.736 eram constituídas por equipes modalidade II, sendo que aproximadamente um terço delas (537) estava distribuído pelo Estado de Minas Gerais. A ausência de TSB nas ESB só poderia ser considerada aceitável, se essa fosse uma estratégia de transição, uma vez que a sua presença sempre resulta em um aumento considerável de utilidade no sistema ${ }^{12}$.

O perfil de atuação dos auxiliares em odontologia no mundo é extenso e seus serviços são bem definidos. No entanto, sua utilização nem sempre foi aceita pelos cirurgiões-dentistas brasileiros. Frequentemente houve polêmica sobre suas competências, as funções que lhe devem ser atribuídas e sua formação ${ }^{13,14}$.

Diante do recente marco legal dos TSB no Brasil e da importância de tal profissional para a racionalidade da organização do processo de trabalho, principalmente no Sistema de Único de Saúde do Brasil, é essencial detectar o perfil desses profissionais e sua forma de atuação nos locais onde trabalham. Isso possibilitará verificar se eles executam as atribuições para as quais estão habilitados legalmente, bem como se estão desenvolvendo ações em consonância com a Equipe de Saúde da Família. Estudo anterior realizado com amostra calculada, mas não probabilística, identificou o perfil sociodemográfico e profissional dos THD de Minas Gerais em 1996 ${ }^{15}$. No entanto, até onde foi possível revisar a literatura, nenhum estudo representativo foi realizado para se avaliar o perfil profissional dos TSB em Minas Gerais.

O presente trabalho teve como objetivo analisar o autorrelato de funções executadas pelos Técnicos em Saúde Bucal na Estratégia Saúde da Família no Estado de Minas Gerais.

\section{Métodos}

Foi realizada uma entrevista estruturada com os TSB da ESF do Estado de Minas Gerais, através de um survey telefônico ${ }^{16}$. Os nomes dos TSB e os locais de trabalho dos mesmos foram localizados no sítio do Cadastro Nacional de Estabelecimentos da Saúde (CNES), onde se obteve, também, o número de equipes odontológicas 
com TSB que estavam registradas na ESF do Estado de Minas Gerais, para posteriormente ser calculado o tamanho da amostra.

Um questionário foi construído com base em um instrumento proposto por Queluz ${ }^{17}$ ao analisar o perfil das ocupações auxiliares da odontologia e suas implicações no mercado de trabalho. O questionário foi adaptado segundo o objetivo desta pesquisa e de acordo com as atribuições estabelecidas pela lei $11.889^{6}$. Para a avaliação da confiabilidade do instrumento proposto, foi realizado o teste-reteste do mesmo por meio de telefone, com 20 TSB da ESF de Minas Gerais selecionadas de forma aleatória simples ${ }^{18}$. A concordância foi estimada pela estatística kappa de Cohen (variáveis categóricas) e pelo Coeficiente de Correlação Intraclasse - ICC (variáveis quantitativas), usando o programa SPSS for Windows, versão 17.0. Valores de coeficiente kappa superiores a 0,60 foram considerados adequados. Em apenas 5\% das questões, o kappa foi inferior a 0,60 . Questões que obtiveram uma confiabilidade baixa (kappa menor do que 0,60 ) foram reformuladas e testadas novamente com 12 novos TSB selecionados de forma aleatória simples. Feito isso, os dados foram analisados novamente, e obteve-se um kappa maior do que 0,60 em todas as questões analisadas. As variáveis quantitativas alcançaram valores de ICC entre 0,99 e 1, não sendo necessária, portanto, a realização de nova medição. Sendo assim, o processo de avaliação do questionário foi adequado para as características investigadas.

A aplicação dos questionários foi realizada por telefone. Cada TSB respondeu ao questionário na sua própria unidade de saúde ou no local que considerasse mais conveniente e obedecendo ao dia e ao horário mais favorável. No início das entrevistas, os sujeitos foram esclarecidos sobre o objetivo do trabalho, e foi lido o termo de consentimento livre e esclarecido pela entrevistadora.

O universo da pesquisa $(\mathrm{N}=545)$ foi igual ao número de equipes odontológicas com TSB na ESF de Minas Gerais, fornecido pelo CNES em maio de 2010. Desse total, foram excluídos os TSB com nomes repetidos e aqueles selecionados para a realização do estudo piloto. Dessa forma, o universo finito de TSB foi igual a 484 .

O cálculo amostral foi realizado considerando uma frequência esperada de $50 \%$, intervalo de confiança igual a $95 \%$, precisão de $5 \%$ e o universo finito de TSB igual a 484. A amostra foi de 231 TSB, sendo, o sorteio dos indivíduos, realizado de forma aleatória simples.
Dos 231 TSB que constituíam a amostra inicial, 27 foram perdidos. Os motivos das perdas foram: o TSB que não trabalhava mais na ESF $(\mathrm{n}=12)$; recusa em participar da pesquisa $(\mathrm{n}=$ 4); e impossibilidade de contato $(n=11)$. Dessa forma, a amostra final foi constituída por 204 TSB. A precisão recalculada foi igual a 5,21\%.

Os resultados da pesquisa foram armazenados pelo programa SPSS for Windows, versão 17.0, e posteriormente analisados por meio de estatística descritiva, com cálculo de proporções e respectivos intervalos com 95\% de confiança (IC 95\%).

O trabalho foi aprovado pelo Comitê de Ética em Pesquisa da Universidade Federal de Minas Gerais. Os princípios éticos foram assegurados, recorrendo-se ao Termo de Consentimento Livre Esclarecido.

\section{Resultados}

A maioria dos TSB é do sexo feminino, representando 97,1\% do total (IC 95\% 93,4\%-98,8\%). A idade média foi de 37,14 anos $( \pm 8,54)$, com o IC 95\% variando entre 36,3 e 38,0 anos.

A descrição das funções executadas pelos TSB inseridos na ESF de Minas Gerais foi agrupada em três categorias: ações assistenciais (Tabela 1); ações preventivas/coletivas (Tabela 2); ações administrativas/organizacionais (Tabela 3 ).

\section{Discussão}

Até onde foi possível revisar a literatura, considera-se que este estudo foi o primeiro representativo de um estado brasileiro. Considerando que Minas Gerais abarcava, aproximadamente, um terço das Equipes Modalidade II do Brasil, sendo o estado com mais TSB na ESF no Brasil ${ }^{19}$, os resultados apresentam maior importância enquanto diagnóstico populacional.

Para melhor compreensão e discussão das atribuições desempenhadas pelos TSB, este estudo dividiu as funções especificadas na lei $11.889^{6}$ de 2008 e que compuseram o questionário aplicado aos TSB em: ações assistenciais, ações preventivas/coletivas e ações administrativas/organizacionais ${ }^{5,17}$.

Os resultados apresentados revelaram que os TSB de Minas Gerais executavam, com maior frequência, ações preventivas/coletivas do que aquelas assistenciais.

No que concerne às atividades clínicas desempenhadas pelos TSB, é importante destacar que 


\begin{tabular}{|c|c|c|c|}
\hline \multirow[t]{16}{*}{ 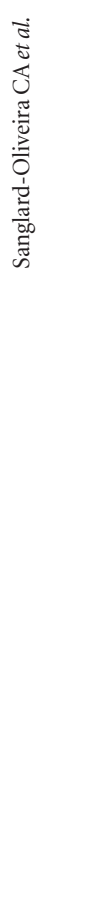 } & Tabela 1. Distribuição das ações assistenciais executadas pelos TSB da ESF, & Minas Gerais, 201 & \\
\hline & Atribuições clínicas & Frequência (\%) & IC 95\% \\
\hline & Faz polimento coronário & 71,6 & $64,4-77,5$ \\
\hline & Faz raspagem de tártaro & 63,2 & $56,1-69,7$ \\
\hline & Tira radiografias intraorais & 13,7 & $9,4-19,3$ \\
\hline & Revela radiografias intraorais & 17,1 & $12,8-23,7$ \\
\hline & Cartona radiografias intraorais & 18,1 & $13,2-24,2$ \\
\hline & Insere e condensa substâncias restauradoras & 14,7 & $10,3-20,4$ \\
\hline & Faz limpeza e antissepsia do campo operatório, antes e após atos cirúrgicos & 56,4 & $49,2-63,2$ \\
\hline & Remove sutura & 80,4 & $74,1-85,4$ \\
\hline & Promove isolamento do campo operatório & 8,3 & $5,0-13,2$ \\
\hline & Prepara o paciente para o atendimento & 83,8 & $77,8-88,4$ \\
\hline & Auxilia e instrumenta os profissionais no atendimento clínico & 45,1 & $38,1-52,1$ \\
\hline & Manipula materiais de uso odontológico & 46,6 & $39,6-53,6$ \\
\hline & Seleciona moldeiras & 7,8 & $4,6-12,6$ \\
\hline & Confecciona modelos de gesso & 7,4 & $4,3-12,0$ \\
\hline
\end{tabular}

Tabela 2. Distribuição das ações preventivas/coletivas executadas pelos TSB da ESF, Minas Gerais, 2010.

\begin{tabular}{lcc}
\hline \multicolumn{1}{c}{ Atribuições preventivas/coletivas } & Frequência (\%) & \multicolumn{1}{c}{ IC 95\% } \\
\hline Participa na realização de levantamento epidemiológico & 89,2 & $83,9-92,9$ \\
Participa de ações educativas, atuando na promoção de saúde e na & 100,0 & $97,6-100,0$ \\
prevenção das doenças bucais & & \\
Faz demonstração de técnicas de higiene bucal (escovação, etc.) & 99,0 & $96,1-99,8$ \\
Faz aplicação tópica de flúor & 96,6 & $92,7-98,4$ \\
Realiza visitas domiciliares junto com a ESF & 77,9 & $71,5-83,3$ \\
Realiza atividades preventivas/educativas nas visitas domiciliares & 77,5 & $70,9-82,8$ \\
Realiza atividades clínicas nas visitas domiciliares & 15,2 & $10,7-21,0$ \\
Realiza levantamento epidemiológico nas visitas & 32,4 & $26,0-39,3$ \\
Realiza ações coletivas em creches & 70,6 & $63,7-76,6$ \\
Realiza ações coletivas em escolas & 96,6 & $92,7-98,4$ \\
Realiza ações coletivas em outros locais & 72,5 & $65,8-78,4$ \\
Participa de reuniões e planejamento juntamente com a equipe da ESF & 75,5 & $68,8-81,1$ \\
\end{tabular}

Tabela 3. Distribuição das ações administrativas/organizacionais executadas pelos TSB da ESF, Minas Gerais, 2010.

\begin{tabular}{lcc}
\hline \multicolumn{1}{c}{ Atribuições administrativas/organizacionais } & Frequência (\%) & IC 95\% \\
\hline Marca consulta & 82,4 & $76,2-87,1$ \\
Lava os instrumentais & 52,5 & $46,3-59,4$ \\
Esteriliza os instrumentais & 44,6 & $37,7-51,7$ \\
Faz conservação e manutenção de equipamentos odontológicos & 87,3 & $81,7-91,3$ \\
Aplica medidas de biossegurança quando armazena, manuseia e descarta & 98,5 & $95,4-99,6$ \\
os produtos e resíduos odontológicos & & \\
Faz limpeza do consultório (passar pano, etc.) & 14,7 & $10,3-20,4$ \\
Realiza o acolhimento do paciente nos serviços de saúde bucal & 93,1 & $88,5-96,0$ \\
\hline
\end{tabular}


a maioria realizava o polimento coronário e a raspagem de tártaro supragengival. Em um estudo realizado por Queluz ${ }^{17}$, antes mesmo da legislação de 2008, apenas $25,5 \%$ dos técnicos realizavam a remoção de restos alimentares, placas e cálculos supragengivais, resultados inferiores aos obtidos neste estudo. A lei estabelece que os TSB têm a atribuição de "remover o biofilme", não especificando, entretanto, a consistência deste na cavidade bucal, mineralizado ou não. O cálculo dentário é um tipo de depósito incluído entre os tipos de biofilme. Não há qualquer disposição na lei $11.889^{6}$ que coíba a realização da raspagem de cálculo dentário pelo TSB no contexto do processo de trabalho de uma Equipe de Saúde Bucal. A interpretação de que, com a Lei, o TSB não poderia mais remover cálculo dentário é equivocada, sendo que tal atribuição já era bem consolidada nas regulamentações dos TSB há anos. Assim, não se sustenta impedir que um TSB realizasse tal procedimento sob a alegação de que a lei não permita.

A partir da experiência de Dunning ${ }^{13}$, confirmada em dezenas de outras posteriores ao longo das décadas, considera-se que o técnico ideal seja aquele que está habilitado para os procedimentos de inserção de materiais em cavidades dentais previamente preparadas por cirurgiões-dentistas, e que realize condensação, escultura, acabamento e polimento das restaurações. A ausência de qualquer etapa do procedimento restaurador citado implica em debilitação técnica, o que retiraria do TSB brasileiro representatividade para comparação com os estudos consagrados internacionalmente. De acordo com a legislação atual, o TSB pode fazer apenas a inserção e condensação de materiais restauradores em cavidades preparadas pelo cirurgião-dentista. Assim, há uma brusca interrupção no trabalho da restauração dentária. Essa fragmentação caracteriza-se pela impossibilidade de se finalizar um procedimento restaurador, com a realização da escultura. Essa fragmentação pode levar a uma diminuição das ações clínicas do TSB com o usuário. Nesse último caso, parece haver a defesa de interesses corporativistas de décadas atrás. Isso pode ser visto, neste trabalho, através da baixa porcentagem de TSB que relataram fazer a inserção e a condensação de substâncias restauradoras (14,7\%). Essa é a única atividade delegada ao TSB que se assemelha às atividades operatórias das terapeutas dentais da Nova Zelândia ${ }^{1}$. Esse fato confirma que, ao se aprovar a lei $11.889^{6}$, delegou-se aos TSB funções bem mais limitadas, talvez por cautela e medo da reação corporativista odontológica. Por isso, também, a lei $11.889^{6}$ especifica que os TSB podem atuar somente com a supervisão do cirurgião-dentista e não podem trabalhar de forma autônoma como na Suécia, Dinamarca e no Estado do Colorado $(\text { EUA })^{4}$. Pesquisa realizada com algumas ESB da Prefeitura de Belo Horizonte, revelou que os cirurgiões-dentistas consideraram o trabalho clínico da TSB como bastante satisfatório. A ausência de realização de procedimentos restauradores por THD diminui a oferta assistencial em saúde bucal de 30 a $50 \%{ }^{20}$.

A baixa porcentagem de TSB que realizavam tomadas radiográficas $(13,7 \%)$, que faziam isolamento do campo operatório $(8,3 \%)$ e que confeccionavam modelos de gesso $(7,4 \%)$ pode ser um reflexo, também, da ausência dessas tecnologias nos serviços públicos brasileiros de saúde bucal ${ }^{17}$.

Além dos procedimentos clínicos descritos acima, os TSB também são profissionais com funções importantes no Global Oral Health Programme, programa da Organização Mundial de Saúde que visa atingir os objetivos mundiais para a saúde bucal em 2020, com especial enfoque na modificação dos comportamentos de risco, através da prevenção das doenças bucais e da promoção dos conhecimentos e práticas de saúde ${ }^{7}$. Vários trabalhos internacionais relatam a importância dos higienistas dentais, cuja ocupação tem perfil semelhante ao dos TSB, para a promoção e a educação em saúde $e^{4,5,21,22}$.

A lei $11.889^{6}$ deixa explícitas que os TSB têm amplas funções em relação aos procedimentos coletivos. Sob a frase "participar das ações educativas atuando na promoção da saúde e na prevenção das doenças bucais", são criadas condições de atuação do TSB como multiplicador e até mesmo como supervisor. Neste caso, não se impõe vedações específicas, apesar de se expor uma supervisão indireta dos TSB por parte do cirurgião-dentista. Essa atuação do TSB na promoção de saúde, com a identificação de riscos comuns tanto à saúde bucal, quanto à saúde geral, constitui um grande ganho da legislação, o que não se percebia nas normatizações anteriores ${ }^{5}$.

A partir dos resultados deste estudo, podemos perceber que os TSB têm se direcionado cada vez mais para a execução de tarefas baseadas na concepção da promoção de saúde, que é fundamental na Atenção Primária à Saúde ${ }^{9}$ e que rege o modelo de atenção da ESF adotado no Brasil $^{10,11}$. Resultados semelhantes foram encontrados em outros estudos realizados nos programas de saúde pública dos Estados Unidos e do Brasil $^{15,23,24}$. Todos os TSB da presente pesquisa rela- 
taram que participavam de ações educativas, atuando na promoção de saúde e na prevenção de doenças bucais, e quase todos faziam a demonstração de técnicas de higiene bucal. Os espaços coletivos onde eram realizadas essas atividades envolviam, principalmente, escolas (96,6\%), confirmando os dados observados por Frazão ${ }^{24} \mathrm{dez}$ anos antes da aprovação da lei. Outros locais em que as ações coletivas são desenvolvidas pelos TSB demonstram que, em muitos centros de saúde, existe uma interação entre as ESB e as Equipes de Saúde da Família, o que é preconizado pela atual Política Nacional de Saúde Bucal. A sua participação nas reuniões junto com a equipe da ESF $(75,5 \%)$ indica, também, essa interação, apesar de evidências anteriores apontarem para a necessidade de maior envolvimento ${ }^{25}$. Esse distanciamento ainda existente em algumas equipes, talvez, possa ser explicado por alguns fatores como a inserção tardia das ESB na ESF e a formação excessivamente individualista e tecnicista dos profissionais de saúde bucal, o que dificulta a abordagem do indivíduo como um todo.

A partir da reorganização das práticas de saúde bucal baseadas na Atenção Primária, o técnico representa um potente e valorizado componente de uma ESB atuando na comunidade. Esse perfil ampliado de suas atribuições, descritas na lei $11.889^{6}$, que cita pela primeira vez a atuação do TSB na promoção de saúde, delineia com nitidez que o TSB é mais um agente multiplicador e educador de saúde, com intervenção direta extraclínica, participando e colaborando na programação das visitas nos domicílios e em ações coletivas de saúde.

Por outro lado, é importante discutir que essa maior valorização dada às ações promocionais nos espaços clínicos e coletivos poderia, também, ser delegada a Agentes Comunitários de Saúde (ACS) e multiplicadores devidamente qualificados, profissionais que integram as Equipes de Saúde da Família.

Segundo a lei $11.889^{6}$, todas as funções delegadas aos Auxiliares de Saúde Bucal (ASB) são também atribuídas aos TSB. Assim, algumas funções administrativas/organizacionais, como a marcação de consulta $(82,4 \%)$, conservação e manutenção de equipamentos odontológicos $(87,3 \%)$ e o acolhimento do paciente $(93,1 \%)$, foram relatadas pela maior parte dos TSB entrevistados. Essa última conduta citada, "acolher o paciente", constitui uma das diretrizes da Política Nacional de Humanização ${ }^{26}$.

Outras funções administrativas/organizacionais foram realizadas pelos TSB em menor fre- quência, como a lavagem (52,5\%) e a esterilização (44,6\%) dos instrumentais, porém em frequência superior àquela identificada em estudo anterior ${ }^{25}$. A instrumentalização do cirurgião-dentista foi relatada por $45,1 \%$ dos TSB deste estudo, corroborando os resultados encontrados em outro ${ }^{17}$. Essas atribuições poderiam ser desempenhadas mais frequentemente pelos ASB, permitindo que os TSB realizem atividades exclusivas do seu campo profissional. Verificou-se, no presente estudo, uma preocupante subutilização dos TSB, com potencial impacto negativo sobre a organização do cuidado em saúde bucal ${ }^{12}$.

Então, sob o ponto de vista da universalização do acesso e utilização dos serviços de saúde e integralidade, o papel legal do TSB seria fora da clínica, desenvolvendo ações de promoção de saúde. $\mathrm{Na}$ assistência individual, não fossem os limites da lei, ele poderia cumprir também seu papel de operador, seja realizando os procedimentos restauradores, seja executando os procedimentos básicos preventivos de periodontia.

Atualmente, um dos importantes impulsionadores da formação de Técnicos em Saúde Bucal no Brasil é a Política Nacional de Saúde Bucal. Os TSB inseridos nas Equipes de Saúde Bucal buscam consolidar a prática em saúde voltada para a humanização da atenção, tendo o cuidado e o vínculo como eixo de suas ações. Assim, muito além de atuações clínicas, os TSB têm a função de participar do processo de planejamento, acompanhamento e avaliação das ações desenvolvidas no território de abrangência das unidades básicas de saúde da família, identificar as necessidades e as expectativas da população em relação à saúde bucal, estimular e executar medidas de promoção da saúde, atividades educativas e preventivas em saúde bucal, organizar o processo de trabalho de acordo com as diretrizes do SUS, sensibilizar as famílias para a importância da saúde bucal na manutenção da saúde, programar e realizar visitas domiciliares de acordo com as necessidades identificadas e desenvolver ações intersetoriais para a promoção da saúde bucal ${ }^{27}$.

É importante considerar, entretanto, que o presente estudo avaliou o autorrelato de prática de TSB. O diagnóstico coletivo em saúde pode ser desenvolvido por metodologias quantitativas e/ ou qualitativas. A opção metodológica do presente estudo pelo referencial teórico quantitativo, não desconsidera a necessidade premente de estudos qualitativos, como por exemplo, a pesquisa-ação ${ }^{20}$. Este método permite que se avalie o processo de trabalho com toda sua complexidade. No entanto, métodos quantitativos são úteis 
para se abarcar um fenômeno em abrangência e representativa probabilística. Outros levantamentos em nível nacional, também são importantes. Surveys telefônicos podem levar a resultados diferentes do que outros métodos, no entanto apresentam-se como métodos válidos e com boa viabilidade, especialmente para avaliação de serviços de saúde. No presente estudo, o método apresentou adequada reprodutibilidade e é, conforme comentado anteriormente, considerado válido por diversos estudos realizados no campo da saúde pública e das ciências sociais ${ }^{28-33}$.

Os TSB inseridos na ESF do Estado de Minas Gerais executavam as atribuições clínicas, preventivas/coletivas e administrativas/organizacionais descritas na Lei $11.889^{6}$. Nesse contexto, destacam-se as funções preventivas/coletivas, por serem realizadas sempre em maior frequência do que as demais, o que demonstra que há uma tendência dos TSB, pelo menos no serviço público de Minas Gerais, de atuarem mais nas ações de promoção de saúde, havendo uma menor realização de atividades clínicas individuais.

\section{Colaboradores}

CA Sanglard-Oliveira foi responsável pela coleta e análise de dados e redação do artigo. MAF Werneck contribuiu substancialmente para a construção do referencial teórico e na leitura crítica do artigo científico. SD Lucas e MHNG Abreu foram orientadores do trabalho, contribuindo com a construção do referencial teórico-metodológico, interpretação dos resultados e redação do artigo. Todos os autores revisaram e aprovaram a versão final do artigo.

\section{Agradecimentos}

Este trabalho foi financiado pela Fundação de Amparo à Pesquisa do Estado de Minas Gerais. 


\section{Referências}

1. Carvalho CL. Trabalho e profissionalização das categorias auxiliares em odontologia. Ação Colet 1999; 2(1):25-33.

2. Baltutis L, Gussy M, Morgan M. The role of the dental hygienist in the public health sector: an Australian perspective. Int Dent $J$ 2000; 50(1):29-35.

3. Hopcraft M, McNally C, Ng C, Pek L, Pham T, Phoon W, Poursoltan P, Yu W. Attitudes of the Victorian oral health workforce to the employment and scope of practice of dental hygienists. Aust Dent $J$ 2008; 53(1):67-73.

4. House DR. The efficient use of hygienist: how is the public best served. J Public Health Dent 1987; 47(3):153-154.

5. Frazão P, Narvai PC. Lei nº11.889/2008: avanço ou retrocesso nas competências do técnico em saúde bucal? Trab Educ Saúde 2011; 9(1):109-123.

6. Brasil. Lei no 11.889 de 24 de dezembro de 2008 . Regulamenta o exercício das profissões de Técnico em Saúde Bucal - TSB e de Auxiliar em Saúde Bucal - ASB. Diário Oficial da União 2008; 26 dez.

7. Sanglard-Oliveira CA, Werneck MA, Lucas SD, Abreu MH. Exploring professionalization among Brazilian oral health technicians. Hum Resour Health 2012; 10(1):5.

8. Cornwall A, Shankland A. Engaging citizens: lessons from building Brazil's national health system. Soc Sci Med 2008; 66(10):2173-2184.

9. Starfield B, Shi L. Policy relevant determinants of health: an international perspective. Health Policy 2002; 60(3):201-218

10. Paim J, Travassos C, Almeida C, Bahia L, Macinko J. The Brazilian health system: history, advances, and challenges. Lancet 2011; 377(9779):1778-1797.

11. Pucca J. A política nacional de saúde como demanda social. Cienc Saude Colet 2006; 11(1):243-246.

12. Zanetti CHG. Pesquisa razões práticas: utilidade e justiça na alocação do Técnico de Higiene Dental (THD) para a oferta assistencial programada do PSF (aferindo a validade externa do instrumento de pesquisa). Brasília; UNB, CEAM, NESP, ObervaRH; 2010.

13. Dunning J. Extending the field for dental auxiliary personnel in the United States. Am J Public Health 1958; 48(8):1059-1064.

14. Luciak-Donsberger C. Origins and benefits of dental hygiene practice in Europe. Int J Dent Hyg 2003; 1(1):29-42.

15. Ribeiro ES, Fischer GE, Marques MCM. Perfil do técnico em higiene dental em Minas Gerais. Rev CROMG 1999; 5(3):164-171.

16. Waldman EA, Novaes, HMD, Alburqueque MFM. Inquéritos Populacionais; aspectos metodológicos, operacionais e éticos. Rev Bras Epidemiol 2008; 11(Supl. 1):168-179.

17. Queluz DP. Perfil dos profissionais auxiliares da odontologia e suas implicações no mercado de trabalho. Rev Odont Cienc 2005; 20(49):270-280.

18. Griep RH, Chor D, Faerstein E, Lopes C. Apoio Social: Confiabilidade teste-reteste na escala do Estudo Pró Saúde. Rev Saude Publica 2003; 19(2):625-634.

19. Brasil. Ministério da Saúde (MS). Departamento de Atenção Básica - DAB. 2011. [acessado 2011 maio 13]. Disponível em: http://dab.saude.gov.br/cnsb/ historico_cobertura_sf.php
20. Sá EMO, Oliveira LA, Melo MB, Pereira NNR, Marques ORA. Avaliação das atividades clínicas do Técnico em Saúde Bucal na rede SUS em Belo Horizonte. Bol Saúde 2010; 24(1):63-73.

21. Ayers K, Meldrum AM, Thomson WM, Newton, JT. The working practices and job satisfaction of dental hygienists in New Zealand. J Public Health Dent 2006; 66(3):186-191.

22. Monajem S. Integration of oral health into primary health care: the role of dental hygienists and the WHO stewardship. Int J Dent Hyg 2006; 4(1):47-51.

23. Deal TS. The role of dental hygienists on state public health programs. J Dent Hyg 1990; 64(6):286-289.

24. Frazão P. A participação do pessoal auxiliar odontológico na promoção da saúde bucal. Rev Odontol Univ São Paulo 1998; 12(4):329-336.

25. Lourenço EC, Silva ACB, Meneghin MC, Pereira AC. A inserção de Equipes de Saúde Bucal no Programa Saúde da Família no Estado de Minas Gerais. Cienc Saude Colet 2009; 14(5):1367-1377.

26. Benevides R, Passos E. A humanização como dimensão pública das políticas de saúde. Cien Saude Colet 2005; 10(3):561-571.

27. Brasil. Ministério da Saúde (MS). Diretrizes da política Nacional de Saúde Bucal. Brasília: MS. 2004. [acessado 2012 abr 25]: [cerca de 16 p.]. Disponível em: http://conselho.saude.gov.br/web_comissoes/ cisb/doc/politica_nacional.pdf

28. Lounds JJ, Borkowski JG, Whitman TL. Centers for the Prevention of Child Neglect. Reliability and validity of the mother-child neglect scale. Child Maltreat 2004; 9(4):371-381.

29. Valle SO, Kuschnir FC, Solé D, Silva MA, Silva RI, Da Cunha AJ. Validity and Reproducibility of the Asthma Core International Study of Asthma and Allergies in Childhood (ISAAC) Written Questionnaire Obtained by Telephone Survey. J Asthma 2012; 49(4):390-394.

30. Moore LV, Diez Roux AV, Franco M. Measuring availability of healthy foods: agreement between directly measured and self-reported data. Am J Epidemiol 2012; 175(10):1037-1044.

31. Erhart M, Wetzel RM, Krügel A, Ravens-Sieberer U. Effects of phone versus mail survey methods on the measurement of health-related quality of life and emotional and behavioural problems in adolescents. BMC Public Health 2009; 9:491.

32. Thomas R, Purdon S. Telephone methods for social surveys. Social Research Uptade [serial on the Internet] 1994 Winter [cited 2012 Jul 16];8. Available from: http://sru.soc.surrey.ac.uk/SRU8.html

33. Kempf AM, Remington PL. New Challenges for Telephone Survey Research in the Twenty-First Century. Annu Rev Public Health 2007; 28:113-126.

Artigo apresentado em 16/10/2012

Aprovado em 23/11/2012

Versão final apresentada em 08/12/2012 


\section{ERRATA}

Errata do artigo Atribuições dos Técnicos em Saúde Bucal na Estratégia Saúde da Família em Minas Gerais, Brasil

$\mathrm{O}$ artigo apresenta erro no nome de um autor

p. 2453

onde se lê:

Carla Aparecida Sanglard ${ }^{1}$

leia-se:

Carla Aparecida Sanglard-Oliveira ${ }^{1}$

p. $2454,2456,2458,2460$, onde se lê:

Sanglard CAO et al.

leia-se:

Sanglard-Oliveira CA et al. 